\title{
„Kausalität ist manchmal verführerisch“
}

\author{
Ein Interview mit Dr. Johannes Lörper
}

\begin{abstract}
Dr. Johannes Lörper hat in Mathematik promoviert, war Vorstand einer großen Versicherungsgruppe und Vorsitzender der Deutschen Aktuarvereinigung (DAV). Im Vorfeld des Weltkongresses der Aktuare in Berlin spricht er mit den Mitteilungen über Sinn und Zweck des Aktuarswesens, die Rolle der Aktuarvereinigung, Biometrie, Karriere und siebenfache Olympiateilnahme.
\end{abstract}

Herr Lörper, Sie sind promovierter Mathematiker und waren Vorsitzender der Deutschen Aktuarvereinigung. Wozu brauchen wir Aktuare?

Keine Versicherung würde ohne Aktuare funktionieren. Die Aktuare nehmen essentielle Aufgaben in den Versicherungen wahr: Sie entwickeln Produkte, berechnen Reserven und sind für das Risikomanagement mitverantwortlich.

War das schon immer so?

Die Rolle der Aktuare in Deutschland hat viel mit der Deregulierung des Versicherungsmarktes zu tun. Bis in die neunziger Jahre haben die Versicherungen im Wesentlichen umgesetzt, was ihnen das Bundesaufsichtsamt für das Versicherungswesen, ein Vorgänger der heutigen BaFin, ins Heft geschrieben hatte. Das änderte sich schlagartig 1994: Plötzlich hatten wir viel mehr Freiheiten, aber auch Verantwortung. Die Aktuare konnten sich selbst überlegen, wie Risiken zu bewerten sind. Ihre eigene Beurteilung und Kreativität war damit viel stärker gefragt. In anderen Ländern wie Großbritannien gehörte das schon lange zur Aufgabe der Aktuare.

Das erinnert an die Deregulierung des Finanzmarktes. Im Finanzbereich hat aber die Krise noch einmal viel verändert. Hat das die Versicherungen nicht betroffen?

Doch, sicherlich! Die große Finanzkrise ab 2001 hat das Denken in allen Finanzinstituten noch einmal grundlegend verändert - auch in den Versicherungen. Es wurde ein Risikomanagement aufgebaut, das diesen Namen wirklich verdient hat. Seitdem setzen wir uns viel stärker mit Modellen auseinander, die das Gesamtrisiko für das Unternehmen betrachten. Damit zog auch sehr viel mehr Mathematik in die Branche ein und der Bedarf nach Aktuaren schnellte in die Höhe. Davon profitiert der gesamte Berufsstand noch immer und durch die Digitalisierung der Versicherungen eröffnen sich derzeit weitere, spannende Aufgabenfelder für die Aktuare. Die DAV hat diese Entwicklung bereits vor Jahren erkannt und entsprechend ihre vereinseigene Aus- und Weiterbildung konsequent um Inhalte zum Thema Big Data erweitert.

Warum braucht man dafür Mathematiker/innen mit einer speziellen Aktuarsausbildung?

Ein Aktuar muss seinen Standpunkt vertreten können, im Zweifelsfall auch gegenüber einem Vorstand. Damit man diese Standfestigkeit hat, muss man sich sowohl auf der Seite der Versicherungswirtschaft als auch mit den mathematischen Modellen auskennen. Zusätzlich zur Mathematik brauchen die Aktuare spezielles VersicherungsKnow-how - also Kenntnisse zu den steuerlichen und rechtlichen Rahmenbedingungen der versicherungstechnischen Risiken. Übrigens sind es nicht nur Mathematiker, die Aktuare werden können. Augenblicklich haben etwas mehr als 9o \% Mathematik oder Wirtschaftsmathematik studiert. Aber ein Studium der Physik, Statistik, Volkswirtschaftslehre oder Informatik ist auch ein guter Ausgangspunkt.

Wie viel Mathematik braucht ein Aktuar wirklich in seinem oder ihrem Berufsleben?

Eigentlich ist es gar nicht so viel, aber es wird immer mehr. Natürlich muss man die Modelle beherrschen. Das ist klar. Aber wichtig ist, dass man darüber hinaus gehen kann. Dass man in der Lage ist, die Abweichungen zwischen den Ergebnissen aus den zahlreichen verwendeten Modellen und der Realität einzuschätzen und die Modelle oder Ergebnisse entsprechend anzupassen.

Welche Rolle spielt die Aktuarvereiningung für den Beruf?

Die Arbeit der DAV hat grob gesprochen zwei Richtungen. Zum einen fördert und stärkt sie durch die Erstellung von Hinweise, Richtlinien und Fachgrundsätze ihre Mitglieder, die sich darauf dann jeweils gegenüber ihrer Geschäftsleitung berufen können. Damit können die Mitglieder einen begründeten aktuariellen Standpunkt besser durchsetzen. Andererseits vertritt sie die Aktuare gegenüber der Branche, der Politik und in der Öffentlichkeit.

Wie schaffen Sie das als Verein?

Das Rückgrat der DAV ist das Ehrenamt. Etwa 6oo Mitglieder sind in der Aus- und Weiterbildung sowie in Arbeitsgruppen und Ausschüssen engagiert und arbeiten damit direkt dem Vorstand zu. Hinzu kommt eine große Zahl an Mitgliedern, die sich im Bereich der Aus- und Weiterbildung von Aktuaren, der Nachwuchsförderung an den Hochschulen, der internationalen Arbeit und in zahlreichen Projekten engagieren.

600 ehrenamtliche Mitglieder! Wie viele Mitglieder haben Sie denn insgesamt? 


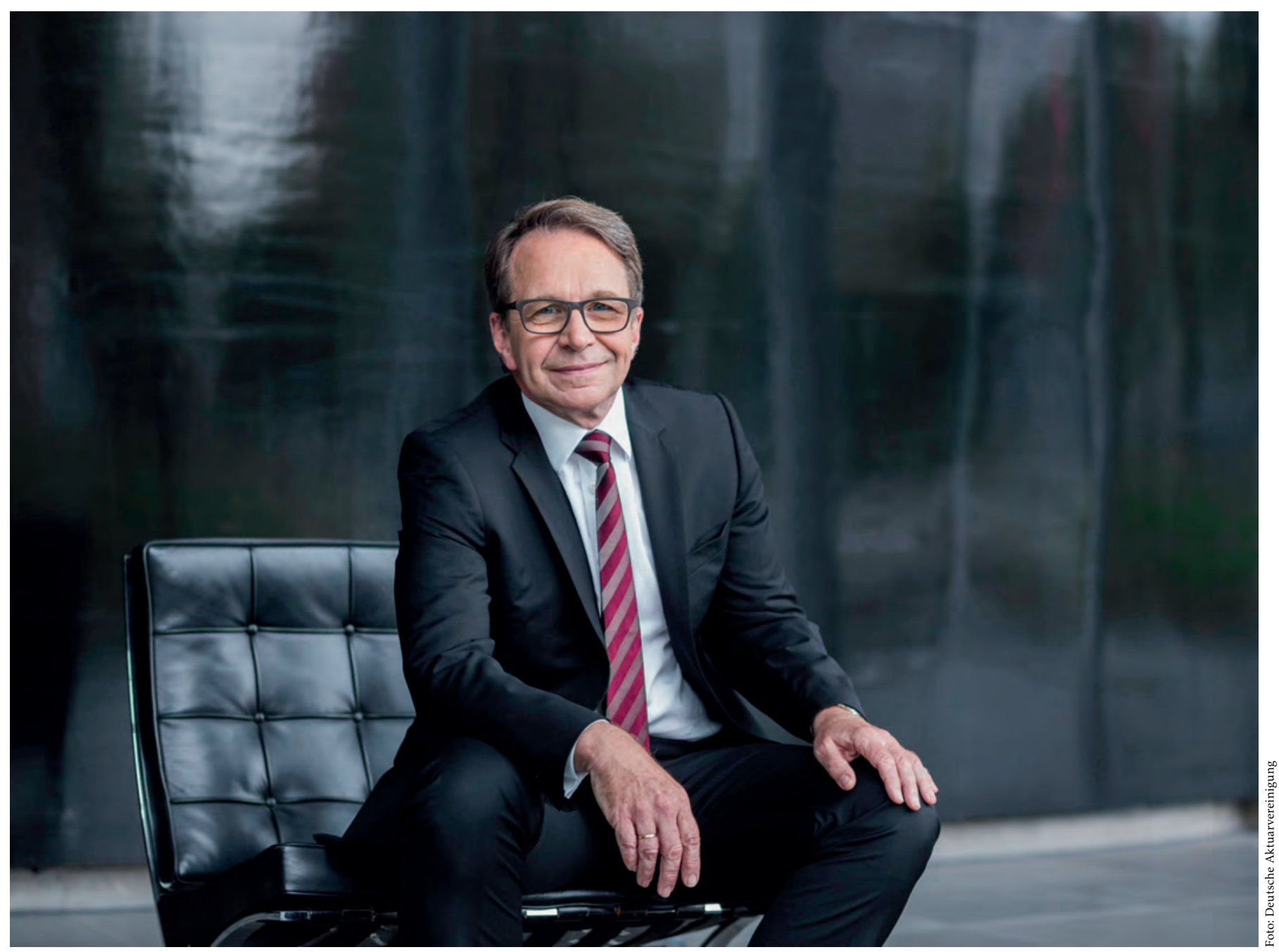

Dr. Johannes Lörper

Wir haben knapp 5200 Mitglieder zuzüglich etwa 1600, die sich in der dreijährigen, berufsbegleitenden Ausbildung zum Aktuar befinden.

Das klingt, als würden alle, die in der Ausbildung zum Aktuar, sind automatisch DAV-Mitglied?

Der Ausdruck „Aktuar“ ist keine geschützte Berufsbezeichnung. „Aktuar DAV“ und „Aktuarin DAV“ allerdings schon. Nicht alle Aktuare in Deutschland sind DAV-Mitglied, aber nahezu alle, sobald sie Mitglied in der DAV sind, verpflichten sich zu gewissen Standesregeln. Dazu gehören zum Beispiel Objektivität, fachliche Unabhängigkeit, Fachkompetenz und Integrität. Es sind auch Mindestanforderungen an die eigenständige berufliche Weiterbildung festgeschrieben. Man muss dokumentieren, dass man sich fachlich auf der Höhe der Zeit hält.

Die DAV akkreditiert auch Kurse, die für die Aktuarsausbildung zugelassen werden sollen, unter anderem auch an Hochschulen. Ein privater, gemeinnütziger Verein der Hochschulen beurteilt. Kommt es da nicht manchmal zu Spannungen?
Ich bin damit selbst nicht so intensiv befasst gewesen. Aber ich hatte immer den Eindruck, dass es einvernehmlich und harmonisch abgelaufen ist.

Intensiv befasst haben Sie sich mit Biometrie. Ich denke da an Gesichtserkennung und Pässe. Aber in der Versicherungswelt meint das etwas anderes.

Unter Biometrie verstehen wir als Versicherer alle Risiken, die mit dem menschlichen Leben einhergehen - also Tod, Krankheit oder Invalidität. Wir beschäftigen uns mit der Frage, wie lange unsere Versicherungskunden leben und wie stark deren Lebenserwartung vom Durchschnittsdeutschen abweicht, um beispielsweise Rentenversicherungsprodukte konstruieren und dafür adäquat Reserven bereit stellen zu können. Es ist enorm wichtig, diese Produkte richtig anzulegen, denn für eine Reihe von Unternehmen ist das der dominierende Geschäftsbereich. Wenn da etwas schiefgeht, lässt sich das nicht durch andere Bereiche ausgleichen. Dann gerät der ganze Tanker in Schieflage.

Sie sprechen von unterschiedlicher Lebenserwartung zwischen dem durchschnittlichen Deutschen und ei- 
nem Besitzer von Rentenversicherungen. Wie kommt es dazu?

Wir sehen zwei Dinge: Zum einen ist die Lebenserwartung beispielsweise mit einem höheren Bildungsstand und einem höheren Einkommen korreliert. Und zum anderen entscheiden sich im gehobenen Alter vor allem die Leute für eine Versicherung mit lebenslanger Rentenzahlung, die sich subjektiv fitter fühlen. Beide Effekte führen dazu, dass die Sterblichkeitsverhältnisse in den Versicherungsbeständen signifikant von denen der allgemeinen Bevölkerung abweichen.

Wie kommen die Versicherungen zu diesen Erkenntnissen?

Durch die Analyse von Daten der Versichertenbestände. Allerdings sind hierzu die wenigsten Versicherer auf Basis ihrer eigenen Daten im Stande, die notwendige Datenbasis für eine sachgerechte Reservierung vorzuhalten. Deshalb hat die Deutsche Aktuarvereinigung als Zusammenschluss der hiesigen Aktuare bereits vor vielen Jahren diese Aufgabe für die gesamte Branche übernommen und erstellt die sogenannten DAV-Sterbetafeln. Die eigentliche Ausgestaltung der Tarife obliegt aber dem jeweiligen Verantwortlichen Aktuar in den Unternehmen, der dabei die firmenindividuellen Verhältnisse zu berücksichtigen hat.

Auf welcher Datenbasis erstellt man die Sterbetafeln?

Für ein möglichst umfassendes Bild beruhen die DAVRententafeln auf einer Vielzahl von Quellen, von denen ein Datenpool mit den Todesfallstatistiken der Versicherungsunternehmen die wichtigste ist. Dank einer breiten Datenzulieferung kann die DAV dabei die anonymisierten Daten von 70 bis 8o Prozent aller deutschen Rentenversicherungsverträge auswerten. Darüber hinaus werden zum Beispiel die Daten zur allgemeinen Bevölkerungssterblichkeit, die jährlich durch das Statistische Bundesamt veröffentlicht werden, sowie die Berechnungen von internationalen Forschungseinrichtungen wie der Human Mortality Database und der Gerontology Research Group zu sogenannten Supercentenarians - Personen über 100 Jahre - sowie Auswertungen der Deutschen Rentenversicherung herangezogen.

Und was machen Sie dann mit den Daten?

Aus den Daten werden rohe Versichertensterblichkeiten berechnet. Das heißt, zum Beispiel im Alter 30 sterben 6o von 100 ooo Versicherungsnehmern. Das ist aber nur der erste Schritt. Tatsächlich verwenden wir keine anzahl-, sondern summengewichtete Sterblichkeiten, da diese mit der Versicherungssumme korreliert sind. Um die Ergebnisse anschließend zu glätten, werden Ausgleichsmethoden wie zum Beispiel das Whittaker-HendersonVerfahren verwendet.

\section{Und dann ist die Arbeit des Aktuars abgeschlossen?}

Nein, natürlich nicht! Denn wir müssen uns stets mit der Frage auseinandersetzen, wie sich die Lebenserwartung künftig entwickeln könnte - welche Trends sind also zu erwarten. Trotz der großen Datenbasis lassen die Versichertendaten hierzu keine zuverlässigen Aussagen zu, da sich die Grundgesamtheit immer wieder verändert oder für einzelne Altersklassen zu wenige Daten vorliegen. Daher verwenden wir für die Herleitung der Trends die zensusbasierten Bevölkerungstafeln. Aber selbst die reichen nicht aus, um belastbare Aussage für die hohen Alter zu treffen. Für die Extrapolation der Ergebnisse in diese hohen Alter verwenden wir internationale Daten zur Sterblichkeit. Zuletzt ist dann noch zu überprüfen, ob die gesamtgesellschaftlichen Trends auf unsere Versicherungskollektive passen. Denn wir wissen zum Beispiel: Beamte leben länger als Angestellte und diese wiederum länger als Arbeiter. Zur Berücksichtigung des Modellrisikos, das insbesondere darin besteht, dass sich die Trends zukünftig anders entwickeln als sie in der Vergangenheit beobachtet wurden, wird von uns ein Sicherheitszuschlag angesetzt. Auch dieser muss noch in die Betrachtung einbezogen werden, bevor wir schlussendlich eine jahrgangsabhängige Sterbewahrscheinlichkeit für jedes einzelne Alter erhalten.

\section{Was sagt denn der Trend? Werden wir immer älter?}

Generell ist erst einmal zu sagen: Seit 175 Jahren haben wir hierzulande einen kontinuierlichen Trend zur Verbesserung der Lebenserwartung, der sich in den vergangenen 25 Jahren im vereinten Deutschland sogar noch einmal beschleunigt hat. So sinkt die Sterbewahrscheinlichkeit inzwischen um zwei bis drei Prozent pro Jahr. Dies hat sich auch in der letzten Überprüfung bestätigt. Dabei zeigte sich wieder einmal eine starke Korrelation zwischen Langlebigkeit und sozioökonomischen Faktoren wie Einkommen, Bildungsstand und Berufswahl. Besonders interessant fanden wir die Ergebnisse der Gerontology Research Group zu den Hochbetagten: Bei der Auswertung der internationalen Studien zu den Supercentenarians war keine signifikante Verbesserung der Lebenserwartung zu erkennen.

Was ist das Besondere an dieser Erkenntnis?

Ich persönlich hätte erwartet, dass wir in dieser Altersgruppe aufgrund des medizinischen Fortschritts deutlich stärkere Trends bei der Lebenserwartung erkennen würden. Das spricht entweder dafür, dass die Menschheit doch so langsam an ihr biologisches Höchstalter herangekommen ist oder dass die Pharma- und Medizinindustrie die Zielgruppe der Hochbetagten noch nicht als Zielgruppe erkannt kann.

Sie betonen das "noch". Glauben Sie, dass sich das ändert?

Wir können es zumindest nicht ausschließen. Denn wenn immer mehr Menschen 90 Jahre und älter werden, dann könnte diese Altersgruppe für die Industrie doch interessant werden und dann könnten sich die Trends beschleunigen. Das müssen wir als Aktuar berücksichtigen und entsprechende Risikopuffer in unsere Berechnungen einfließen lassen. Und das ist genau das vorhin angespro- 


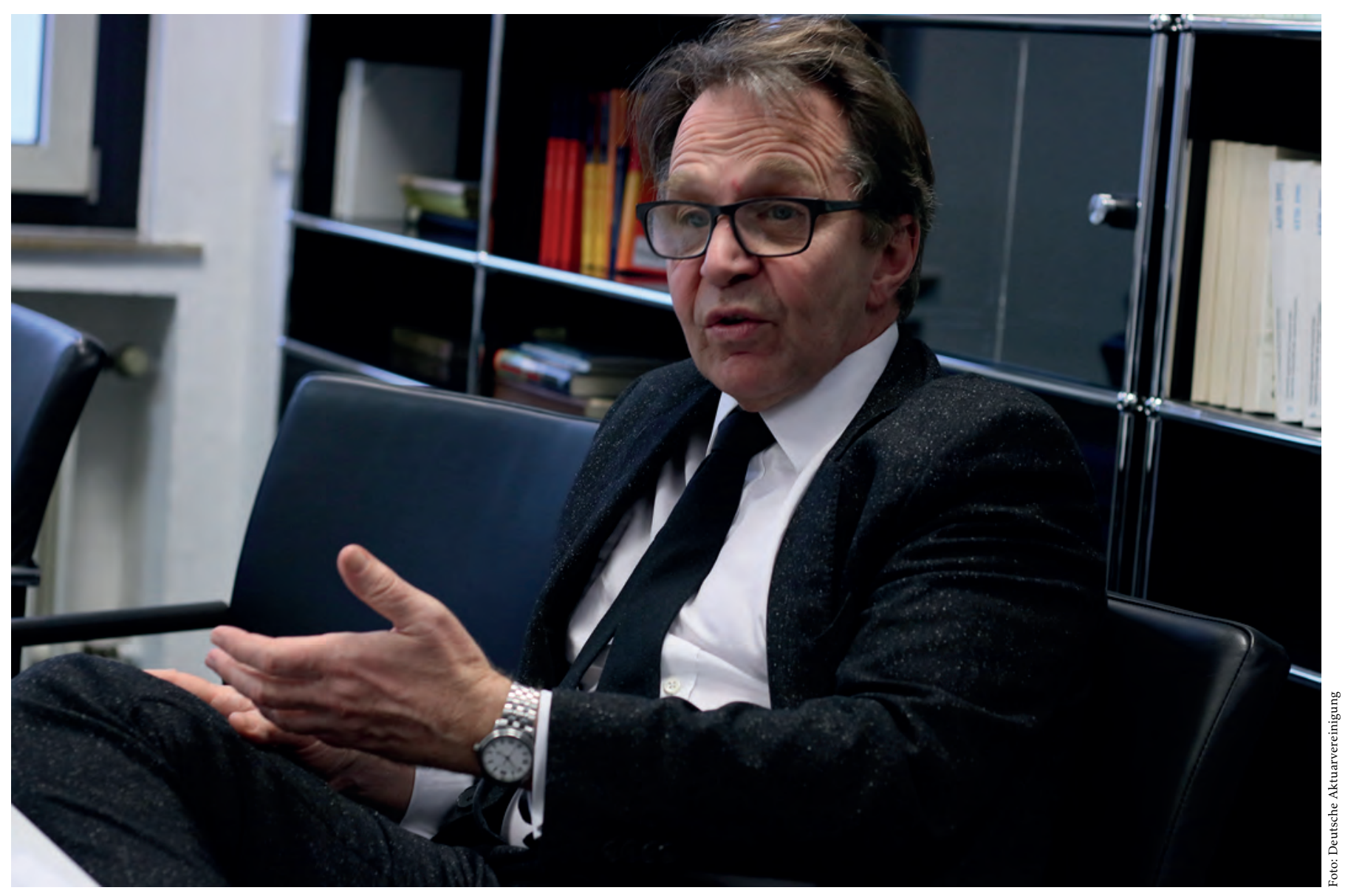

chene Einordnen der Ergebnisse in größere Zusammenhänge.

\section{Das klingt fast spekulativ für einen Mathematiker.}

Nicht unbedingt. Es ist richtig, dass wir mit vielen Unsicherheiten leben müssen, das ist auch Teil unserer Jobbeschreibung. Die Mathematik gibt uns viele Möglichkeiten an die Hand, diese Unsicherheiten zu reduzieren. Aber dann muss der Aktuar auf Grundlage seiner Erfahrung entscheiden, was er mit den vorliegenden Ergebnissen anfängt. Wir Aktuare sind es schlussendlich, die vor der Frage stehen: Sind die vorliegenden Ergebnisse genügend sicher, um darauf aufbauen ein Geschäftsmodell zu entwickeln? Andererseits dürfen wir aber auch nicht zu viele Puffer einbauen, denn die Wettbewerbsfähigkeit muss natürlich gewährleistet sein.

Zuviel Puffer würde doch bedeuten, dass die Produkte für den Kunden zu teuer sind, und die Versicherung sich nachher freuen kann, dass alles doch nicht so schlimm gekommen ist.

Dagegen schützt zum einen der Markt, zum anderen hat aber auch der Gesetzgeber direkt eingegriffen. Zum einen verpflichtet uns der Gesetzgeber, bei der Erstellung der Sterbetafeln besondere Vorsicht walten zu lassen und angemessene Puffer einzurechnen. Denn es wäre katastrophal, wenn wir zu unvorsichtig rechnen. Dann wäre das gesamte System der betrieblichen und privaten Altersvorsorge gefährdet. Andererseits: wenn sich herausstellt, dass wir zu vorsichtig gerechnet haben, dann sieht die Mindestzuführungsverordnung vor, den Kunden mindestens 90 Prozent der Überschüsse auszuzahlen. Das ist in meinen Augen ein fairer Deal.

Sie sagen „in meinen Augen“. Sehen das manche anders?

Es gibt Verbraucherschützer, die auch mit dieser Regelung noch nicht zufrieden sind. Da wird oft jede Art von Puffer in Frage gestellt, oder man sagt, die Rückzahlungen kämen nicht den richtigen zu gute. Ich denke, das geht an der Realität vorbei.

Es gibt eine kritische, öffentliche Diskussion um datengetriebene, letztlich mathematische Methoden in der Versicherungswirtschaft, insbesondere, wenn immer kleinere Datenpunkte aus dem Alltag verarbeitet werden sollen. Verstehen Sie die Furcht, dass Kausalität vollends von Korrelation überschrieben wird?

Die Kausalität steht nicht immer im Mittelpunkt unserer Überlegungen. 
Das müssen Sie mir erklären!

Ich gebe Ihnen ein Beispiel. Die Sterblichkeit der Menschen in Deutschland und anderen Ländern sinkt seit langem. Die Menschen leben länger. Das kann man in vielerlei Hinsicht in vorliegenden Daten beobachten. Nun gab es einen Kollegen in Großbritannien, dem auffiel, dass gleichzeitig der Anteil der Raucher an der Bevölkerung deutlich gesunken war. Also schlug er vor, man solle die Verlängerung der Lebenszeiten, die durch den geringeren Anteil an Rauchern entsteht, herausrechnen. Denn vielleicht werden wir gar nicht älter oder leben gar nicht allgemein gesünder, sondern rauchen einfach weniger. Wir sehen vielleicht in den Zahlen nur den Effekt des Rauchens. Irgendwann, zum Beispiel, wenn fast alle aufgehört haben zu rauchen, fällt dieser Effekt weg und die Prognose, dass wir immer länger leben, stimmt nicht mehr. Also sollte man mit Blick auf diese Kausalität die Prognose rechtzeitig korrigieren.

Das klingt doch sehr vernünftig.

Vielleicht, vielleicht aber auch nicht. Vielleicht ist die Reduktion des Rauchens nur ein erster Schritt eines allgemeinen Trends, gesünder zu leben. Oder vielleicht gibt es in Zukunft eine andere gesellschaftliche Umwälzung oder medizinische oder technische Innovation, die wir uns heute noch nicht vorstellen können - so wie man sich nicht vorstellen konnte, dass so viel weniger Menschen rauchen. Weder die eine noch die andere Annahme für die Zukunft können wir belegen. Deswegen stellen wir uns auf den Standpunkt, wir halten uns an den Trend, so wie er in den Zahlen nachweisbar ist.

In den kommenden Tagen findet der Weltkongress der Aktuare im Juni dieses Jahres in Berlin statt. Wie stehen die DAV im internationalen Vergleich da?

Weltweit zählen die Aktuarvereinigungen etwa 100 ooo Mitglieder. Der Beruf stammt aus dem angelsächsischen Bereich. In den USA gibt es drei Aktuarvereinigungen, in Großbritannien eine, aber sehr große. Zusammen mit Japan stehen wir auf Platz 5.

Wie wichtig ist Ihnen dieser Kongress?

Zunächst einmal freue ich mich auf meinen siebten Weltkongress. Das ist schon ein bisschen wie eine Olympiade nur eben ohne den Wettbewerbsgedanken.

Und man kann öfter teilnehmen!

Und wir sind stolz, dass wir nach 50 Jahren den Weltkongress erstmals wieder nach Deutschland holen konnten und inzwischen mit 2750 Anmeldungen eine Rekordbeteiligung vermelden können. Für viele deutsche Aktuare ist das sicherlich eine einmalige Chance im Berufsleben, den Weltkongress vor der Haustür zu erleben, denn er wandert wie die Olympiade durch die ganze Welt und findet nur alle vier Jahre statt. Inhaltlich erwarte ich mir spannende Impulse für unseren Berufsstand in allen Versicherungsbereichen. Ich selbst werde mit einer Kollegin etwas mehr darüber erzählen, wie wir die bereits beschriebenen Sterbetafeln konstruieren und mit welchen Trends wir in den kommenden Jahren bei der Lebenserwartung rechnen.

Würden Sie sagen, dass Ihnen Ihr Beruf Spaß gemacht hat?

Ja, absolut. Ich bin schon während meiner Promotion zu einem Rückversicherungsunternehmen gegangen. Das war etwas vollkommen Neues. An der Uni in Köln hatte ich mich vor allem mit theoretischen Fragen beschäftigt. Jetzt ging es darum, das erlernte strukturierte Denken zu nutzen, um Geschäftsmodelle zu rechnen bzw. zu bewerten und weltweit mit den Kunden Verhandlungen zu führen. In meinen ersten Jahren betreute ich Märkte in Israel, Griechenland, Zypern, Australien, Asien und Lateinamerika. Die Mischung aus Anwendung der Theorie - oder vereinfacht gesagt der Rechenkünste - und dem ständigen Kontakt mit den Versicherungsunternehmen vor Ort war toll.

Das klingt nach einer sehr geradlinigen Karriere. Haben Sie nie das Bedürfnis gehabt, etwas ganz anderes zu machen?

Nach 20 Jahren Rückversicherungsgeschäft wurde es Zeit für etwas Neues - und inzwischen hatte ich auch den ganzen Globus bereist. Zudem wollte ich mich auf einen Markt konzentrieren und das konnte ich dann als Vorstand bei der Hamburg-Mannheimer. Das war aber nicht nur inhaltlich eine ganz neue berufliche Welt, sondern auch strukturell. Plötzlich waren nicht mehr einige große Erstversicherer meine Kunden, sondern eine Vielzahl an Einzelpersonen und wir hatten eine viel größere aktuarielle Abteilung als beim Rückversicherer. Das war spannend, mit so vielen Mathematikern zusammenzuarbeiten.

Dass ein Mathematiker bei einer Versicherung arbeitet, habe ich schon einmal gehört. Aber warum sind Sie Vorstand in der ERGO Versicherungsgruppe geworden?

Ich habe mich schon immer dafür interessiert, Prozesse zu strukturieren. Wie etwas dann genau ausgerechnet wird, das hat mich schon im Studium nicht so sehr interessiert. Das sollen lieber andere machen. Aber es gibt natürlich nicht den einen Weg, um Vorstand in einem Versicherungsunternehmen zu werden. Was mir sicherlich zu Gute kam, war meine Kommunikationsfreude. Ich habe es schon immer gemocht, Ideen und Menschen zusammenzubringen. Dafür ist eine gute Prise Extrovertiertheit ganz hilfreich. Wer als fachlich guter Mathematiker sein Gegenüber von seinen Ideen und Vorschlägen überzeugen kann, hat überall sehr gute Karrierechancen.

Mit Johannes Lörper sprach Sebastian Stiller. 